\title{
Massive MIMO with Transceiver Hardware Impairments: Performance Analysis and Phase Noise Error Minimization
}

\author{
Parfait I. Tebe ${ }^{1 *}$, Guangjun Wen ${ }^{1}$, Jian $\mathrm{Li}^{1}$, Yongjun Huang ${ }^{1}$, Affum E. Ampoma ${ }^{1}$, \\ and Kwame O. Gyasi ${ }^{1}$ \\ ${ }^{1}$ Center for RFIC and System Technology, School of Information and Communication Engineering, University of \\ Electronic Science and Technology of China \\ No.2006, Xiyuan Avenue, West High-Tech Zone, Chengdu, 611731, P. R. China \\ [e-mail : pariteb@yahoo.fr,wgj@uestc.edu.cn, lj001@uestc.edu.cn, yongjunh@uestc.edu.cn, \\ ampoma.uestc@yahoo.com, kermaabar@yahoo.com] \\ *Corresponding author : Parfait I. Tebe
}

Received July 21, 2018; revised September 21, 2018; accepted November 27, 2018;

published May 31, 2019

\begin{abstract}
In this paper, we investigate the impact of hardware impairments (HWIs) on the performance of a downlink massive MIMO system. We consider a single-cell system with maximum ratio transmission (MRT) as precoding scheme, and with all the HWIs characteristics such as phase noise, distortion noise, and amplified thermal noise. Based on the system model, we derive closed-form expressions for a typical user data rate under two scenarios: when a common local oscillator (CLO) is used at the base station and when separated oscillators (SLOs) are used. We also derive closed-form expressions for the downlink transmit power required for some desired per-user data rate under each scenario. Compared to the conventional system with ideal transceiver hardware, our results show that impairments of hardware make a finite upper limit on the user's downlink channel capacity; and as the number of base station antennas grows large, it is only the hardware impairments at the users that mainly limit the capacity. Our results also show that SLOs configuration provides higher data rate than CLO at the price of higher power consumption. An approach to minimize the effect of the hardware impairments on the system performance is also proposed in the paper. In our approach, we show that by reducing the cell size, the effect of accumulated phase noise during channel estimation time is minimized and hence the user capacity is increased, and the downlink transmit power is decreased.
\end{abstract}

Keywords: Massive MIMO, transceiver hardware impairments, channel estimation, cell size, system performance

This work was supported in part by the National Natural Science Foundation of China under project contracts No.61601093, No.61791082, No. 61701116 and No.61371047, in part by Sichuan Provincial Science and Technology Planning Program of China under project contracts No.2016GZ0061, No.2018HH0044 and No.2019YFG0418, in part by Guangdong Provincial Science and Technology Planning Program of China under project contracts No.2015B090909004 and No.2016A010101036, in part by the fundamental research funds for the Central Universities under project contract No.ZYGX2016Z011, and in part by Science and Technology on Electronic Information Control Laboratory. 


\section{Introduction}

The next generation wireless communication networks are expected to provide higher data rate with lower energy consumption, compared to the current networks. Massive MIMO technology has been approved to meet such requirements. In the technology, a large number of antennas are deployed at the base stations (BSs) to serve many single-antenna user terminals [1, 2]. It has the potentials of enhancing spectral and energy efficiency in a larger scale compared to conventional MIMO [1-7]. Moreover, when the number of antennas is larger than or equal to a given threshold, the irregular antenna arrays usually used outperform the regular arrays in the achievable rate [8]. The system performance can be further improved with the deployment and application of multiuser detection and beamforming techniques [9]. New detection methods have been also proposed to provide a balanced tradeoff between computational complexity and performance in the system [10]. Furthermore, with the deployment of relay stations, the transmit powers of each source and relay station can be significantly scaled down proportionally to the number of antennas when the number of antennas of the transmit and receive arrays at the relay station is sufficiently large [11].

Despite its potential of being a promising technology, massive MIMO has some limiting factors that affect its performance. Pilot contamination and transceiver hardware impairments are the main limiting factors. Pilot contamination occurs from the use of the same pilot sequences in different co-channel cells and makes the channel state information imperfect [12-15]. Transceiver hardware impairments arise from the antennas involved in the system, its effects lead to errors in estimating the channels and contribute further to the imperfection of the channel state information $[12,16,17]$.

Several research works on factors affecting the performance of massive MIMO have been provided in the literature, but little attention has so far been paid to techniques to mitigate the effect of the transceiver hardware impairments. In [18], the effect of hardware impairments (HWIs) on full-duplex massive MIMO relaying was analyzed. Authors in [19] analyzed different HWIs with regards to the aggregate impact it has on the system. The impact of HWIs with regard to the number of local oscillators used at the base station is analyzed in [20] and [21]. HWIs are modeled as oscillator phase noise in systems studied in [22]. In [23], the authors investigated the impact of HWIs on the downlink system performance. The effect of phase noise on a downlink system is analyzed in [24]. In that work, the authors analyzed and compared the degradation in terms of signal-to-interference-plus-noise ratio and the achievable rate between zero-forcing (ZF) and maximum ratio transmission (MRT) precoders. In [25], the impact of channel aging caused by HWIs is investigated for a downlink system. Authors in [26] investigated the impact of HWIs on the secrecy performance of a downlink system, and their proposed solution mainly focuses on the mitigation of pilot orthogonality loss from phase noise. Authors in [27] used the rate-splitting concept to analyze the performance of massive multiple-input single-output broadcast channel with residual transceiver hardware impairments. In [28], the authors investigate the capacity of a multiple-antenna base station communication system with a single-antenna user over a channel impaired by phase noise, in both uplink and downlink.

Although the aforementioned works consider the problem of hardware impairments in massive MIMO technology, most of them do not provide methods to address the problem but provide analysis on the impact it has on the system performance. The few solution approaches proposed cannot completely remove the HWIs because the techniques and algorithms used do not address all the characteristics of the impairments [20, 23, 25, 26]. Moreover, those methods involve an increase in the system power consumption [20,23] and more complex 
precoding scheme at the base station [26]. In [27] where all the characteristics of the impairments have been taken into consideration, the proposed solution is based on the analysis of the robustness of the rate-splitting concept to each separate hardware imperfection. However, the technique used requires extra precoding and decoding and makes the system more computationally complex.

In this paper, we provide a performance analysis of massive MIMO with transceiver HWIs and propose an approach to minimize the effect of the impairments. We focus on a single-cell downlink system with maximum ratio transmission(MRT) as precoding scheme, and with all the HWIs characteristics [19, 20]. Our main contributions are as follows.

First, we derive closed-form expressions for a typical user data rate under two scenarios: when a common local oscillator(CLO) is used at the BS and when separated oscillators(SLOs) are used. We show that, though the rate increases with the number of base station antennas, there is still some loss due to the transceiver hardware impairments, when compared to the ideal case with no hardware impairments. Moreover, we show that higher data rate can be achieved when SLOs are used; and as the number of base station antennas grows large, it is the users' transceiver hardware impairments that mainly limit the downlink capacity.

Second, we derive closed-form expressions for the downlink transmit power required for some desired per-user data rate under each scenario, and we show that the higher data rate achieved with SLOs configuration is at the price of higher power consumption compared to the use of CLO. We also show for both configurations that, the higher the value of the number of base station antennas or users, the lower the required power.

Third, we propose an approach to minimize the effect of HWIs on the system performance. Our approach relies on reducing the cell size, and we show that when the cell size is reduced the effect of accumulated phase noise during channel estimation time is minimized and hence the user capacity is increased, and the downlink transmit power is decreased.

The difference between our work and [28] which seem to be similar is as follows. [28] considers uplink single-input multiple-output (SIMO) and downlink multiple-input single-output (MISO) with only phase noise as HWI whereas we consider downlink massive multiple-input multiple-output (M MIMO) with all the characteristics of HWIs. [28] focuses only on the analysis of the capacity without any proposed solution to address the problem of phase noise whereas our work analyses the system performance regarding user data rate and required downlink transmit power, with a proposed solution to minimize the effect of phase noise.

The rest of the paper is organized as follows. In Section 2, the system model with hardware impairments is described. Section 3 provides the system performance analysis. Our proposed approach is presented in Section 4. Section 5 is devoted to simulation results, and Section 6 concludes the paper.

\section{System Model with Hardware Impairments}

As shown in Fig. 1, we consider a single-cell system with a Rayleigh fading channel and hardware impairments where $K$ single-antenna users are simultaneously served by $M$ base station antennas. We consider time division duplex (TDD) which is based on channel reciprocity $[2,14]$. In a TDD mode, the transmission time for channel reciprocity between the BS and the terminals within a cell is partitioned into different cycles. The duration of each cycle corresponds to the channel coherence time $T$ with different phases as illustrated in Fig. 2. 


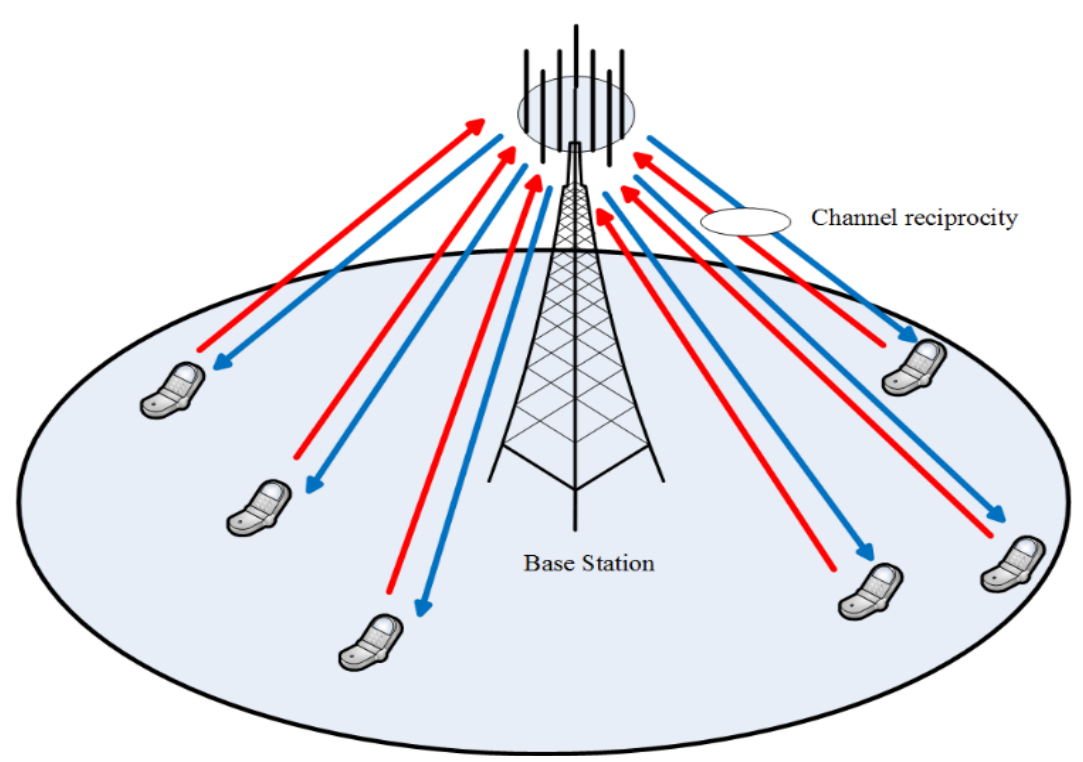

Fig. 1. Illustration of massive MIMO single-cell system

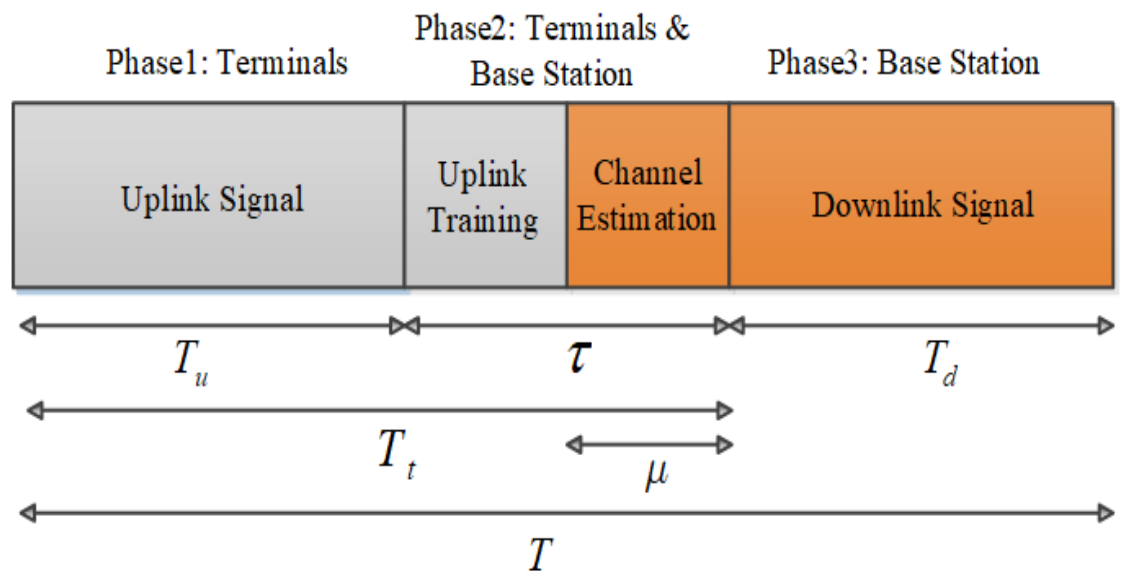

Fig. 2. Transmission cycle in TDD mode

\subsection{Uplink Transmission under Hardware Impairments}

The uplink transmission involves the terminals in phase 1 and phase 2 as shown in the figure, and it is a prerequiste for channel estimation. This is because the channel estimation process needs both users'signals and pilot sequences. Therefore, in phase 1, each terminal transmits signals to the base station in $T_{u}$ time slots; and in phase 2, pilot sequence of length $\tau$ symbols is transmitted by each terminal to the base station. The duration of an uplink transmission cycle within a cell is given as $T_{t}=T_{u}+\tau$, and $\mu=\frac{\tau}{T_{u}+\tau}$ is the fraction of $T_{t}$ devoted for channel estimation [29]. Based on the received signals and pilot sequences, the effective channel is estimated to enable coherent detection of received symbols. We denote $\omega_{k}=$ $\left[\omega_{k}(1), \omega_{k}(2), \ldots, \omega_{k}(\tau)\right]^{T} \in \mathbb{C}^{\tau \times 1}$ the mutually orthogonal pilot sequences emitted by the 
terminals in a set $s_{\tau}$ with $k \in s_{\tau}$. The uplink vector $\boldsymbol{y}^{\mathrm{UL}}(t) \in \mathbb{C}^{M \times 1}$ received at the base station during the uplink transmission is given as [26]

$$
\boldsymbol{y}^{\mathrm{UL}}(t)=\sum_{k \in s_{\tau}} \boldsymbol{\Theta}_{k}(t) \mathbf{h}_{k}\left(\omega_{k}(t)+\eta_{t, k}^{\mathrm{UT}}(t)\right)+\boldsymbol{\eta}_{r}^{\mathrm{BS}}(t)+\zeta^{\mathrm{UL}}(t)
$$

where $\mathbf{h}_{k} \sim \mathbb{C N}\left(\mathbf{0}_{M}, \beta_{k} \mathbf{I}_{M}\right)$ is the channel vector of the $k^{\text {th }}$ user, with $\beta_{k}$ being the path-loss. We thereby assume that $\mathbf{h}_{k}$ is constant during the coherence time $T$ and changes independently afterward [26]. $\boldsymbol{\Theta}_{k}(t)$ is the phase noise, $\eta_{t, k}^{\mathrm{UT}}(t)$ is the distortion noise from user $k, \boldsymbol{\eta}_{r}^{\mathrm{BS}}(t)$ is the distortion noise at the BS, and $\zeta^{\mathrm{UL}}(t)$ is the amplified thermal noise. Those noises are the characteristics of the hardware impairments that affect the uplink training phase.

\subsubsection{Phase Noise}

The phase noise originates from the free-running local oscillator(s) (LOs) at the base station and the users' terminals [21]. In practice, phase noise is induced at the user terminal during up-conversion when the LO carrier is multiplied with the baseband signal. The transmitted signal gets distorted as a result of random drift of the carrier phase and a mismatch between the signal intended to be transmitted and the generated signal. A similar phenomenon occurs at the base station side when the band pass signal is down converted to baseband [25, 27, 30]. The phase noise matrix is given as [26]

$$
\boldsymbol{\Theta}_{k}(t)=\operatorname{diag}\left(e^{j \theta_{k}^{1}(t)} \mathbf{1}_{1 \times M / N_{0}}, \ldots, e^{j \theta_{k}^{M_{0}}(t)} \mathbf{1}_{1 \times M / N_{0}}\right) \in \mathbb{C}^{M \times M}
$$

where $N_{0}$ is the number of LOs; $\theta_{l}^{k}(t)=\psi_{l}(t)+\phi_{k}(t)$ represents the phase noise that affects the link between the $l^{\text {th }} \mathrm{LO}$ at the base station and the $k^{\text {th }}$ user. The phase noise variances at the base station and the mobile users are assumed to be equal and are given as $\sigma_{\psi}^{2}$ and $\sigma_{\phi}^{2}$, respectively [21, 22, 26, 27].

\subsubsection{Distortion Noise}

Distortion noise is an alteration of the signal which occurs during random fluctuations in the electronic circuits. The distortion noises at the user terminal and the BS always arise from residual effects even after compensation efforts of hardware [19, 20]. They are modelled as $\eta_{t, k}^{\mathrm{UT}}(t) \in \mathbb{C}$ and $\boldsymbol{\eta}_{r}^{\mathrm{BS}}(t) \in \mathbb{C}^{M \times 1}$, respectively. The models have been verified in [31] through experiments. Moreover, the distortion noise power has been shown [19] to be proportional to the signal power at each base station. Its distribution is modeled as $\eta_{t, k}^{\mathrm{UT}}(t) \sim \mathbb{C N}\left(\mathbf{0}, v_{t, k}^{\mathrm{UT}}\right)$ and $\boldsymbol{\eta}_{r}^{\mathrm{BS}}(t) \sim \mathbb{C N}\left(\mathbf{0}_{M}, \boldsymbol{\gamma}_{r}^{\mathrm{BS}}\right)$, where

$$
v_{t, k}^{\mathrm{UT}}=\kappa_{t}^{\mathrm{UT}} \mathbb{E}\left[\left|\omega_{k}(t)\right|^{2}\right]
$$

and 


$$
\boldsymbol{\gamma}_{r}^{\mathrm{BS}}=\kappa_{r}^{\mathrm{BS}} \sum_{k=1}^{K} \mathbb{E}\left[\left|\omega_{k}(t)\right|^{2}\right] \mathbf{R}_{k}^{\text {diag }}
$$

Here, $\mathbf{R}_{k}^{\text {diag }}=\operatorname{diag}\left(\left|h_{k}^{1}\right|^{2}, \ldots,\left|h_{k}^{N}\right|^{2}\right)$, with $h_{k}^{i}$ denoting $i^{\text {th }}$ element of $\mathbf{h}_{k} ; \kappa_{t}^{\mathrm{UT}}$ and $\kappa_{r}^{\mathrm{BS}}$ are the proportionality coefficients and are positive.

\subsubsection{Amplified Thermal Noise}

This noise is the amplification of the thermal noise from the amplifiers, mixers and other electronic components at the receiver side and interference leakage from other frequency bands. It is also called receiver noise [20]. Its variance is then larger than that of the actual thermal noise. In the uplink training, this noise is modeled as $\zeta^{\mathrm{UL}}(t) \sim \mathbb{C N}\left(\mathbf{0}_{M}, \zeta^{\mathrm{UL}} \mathbf{I}_{M}\right)$.

\subsection{Channel Estimation and Downlink Transmission under Hardware Impairments}

Channel estimation and downlink transmission involve the base station in phase 2 and phase 3 , respectively, as shown in Fig. 2. In phase 2, the received signal and pilot sequence from each terminal are used to estimate the channel between the given terminal and the base station. In phase 3 , the estimated channel in phase 2 is used by the base station to transmit downlink signals to the terminals in $T_{d}$ time slots. The coherence period of a transmission cycle is given as the sum of the uplink transmission duration and the time slot of the downlink signal transmission as $T=T_{t}+T_{d}$. Assuming that the base station uses minimum mean squared error (MMSE) as channel estimator, for symbol interval $t \in\left\{T_{t}+1, \ldots T\right\}$, the channel estimates of user $k \in s_{\tau}$ is given as [26]

$$
\hat{\mathbf{h}}_{k}(t)=\frac{p_{\tau} \beta_{k} e^{-\frac{\sigma_{\psi+}^{2} \sigma_{\phi}^{2}}{2}|\mu|}}{p_{\tau} \beta_{k}\left(1+\kappa_{t}^{\mathrm{UT}}+\kappa_{r}^{\mathrm{BS}}\right)+\zeta^{\mathrm{UL}}} y^{\mathrm{UL}}\left(T_{t}\right)
$$

where $p_{\tau}$ is the per-pilot power constraint. The corresponding error covariance matrix is given as

$$
\mathbb{E}\left[\boldsymbol{e}_{k}(t) \boldsymbol{e}_{k}^{H}(t)\right]=\beta_{k}\left(1-\frac{p_{\tau} \beta_{k} e^{-\left(\sigma_{\psi+}^{2} \sigma_{\phi}^{2}\right)|\mu|}}{p_{\tau} \beta_{k}\left(1+\kappa_{t}^{\mathrm{UT}}+\kappa_{r}^{\mathrm{BS}}\right)+\zeta^{\mathrm{UL}}}\right) \mathbf{I}_{M}
$$

where $\boldsymbol{e}_{k}(t)$ is the estimation error.

The signal received by the $k^{\text {th }}$ user is given as [26]

$$
\boldsymbol{y}_{k}^{\mathrm{DL}}(t)=\mathbf{h}_{k}^{H} \mathbf{\Theta}_{k}^{H}(t)\left(\mathbf{x}+\boldsymbol{\eta}_{t}^{\mathrm{BS}}(t)\right)+\eta_{r, k}^{\mathrm{UT}}(t)+\zeta_{k}^{\mathrm{DL}}(t)
$$


Similar to the uplink, $\boldsymbol{\eta}_{t}^{\mathrm{BS}}(t) \sim \mathbb{C N}\left(\mathbf{0}_{M}, \boldsymbol{\gamma}_{t}^{\mathrm{BS}}\right)$ and $\eta_{r, k}^{\mathrm{UT}} \sim \mathbb{C N}\left(0, v_{r, k}^{\mathrm{UT}}\right)$ are the downlink distortion noise [20] at the base station and the $k^{\text {th }}$ user, respectively, where

$$
\begin{gathered}
\boldsymbol{\gamma}_{t}^{\mathrm{BS}}=\kappa_{t}^{\mathrm{BS}} \operatorname{diag}\left(X_{11}, \ldots, X_{M M}\right) \text { and } \\
v_{r, k}^{\mathrm{UT}}(t)=\kappa_{r}^{\mathrm{UT}} \mathbf{h}_{k}^{H}(t) \mathbf{X} \mathbf{h}_{k}(t)
\end{gathered}
$$

with $\mathbf{X}=\mathbb{E}\left[\mathbf{x x}^{H}\right]$ and $\mathrm{X}_{i i}=[\mathbf{X}]_{i i}, i=1, \ldots, M$. Furthermore, $\zeta_{k}^{\mathrm{DL}}(t) \sim \mathbb{C N}\left(0, \zeta^{\mathrm{DL}}\right)$ is the amplified thermal noise at the user and $\mathbf{x} \in \mathbb{C}^{M \times 1}$ is the downlink transmit signal.

As explained and shown, various noises from transceiver hardware impairments generate error that affects the channel state information and the system performance. In this paper, we analyze the system performance under HWIs and propose an approach to address the problem. The analysis and approach are provided in the following sections.

\section{Performance Analysis in the Presence of Hardware Impairments}

The system performance is related to the signal-to-interference-plus-noise ratio (SINR), where the interference consists of multiuser interference, and the noise originates from the HWIs at the BS and user terminals. In our analysis, we assume that all the base station antennas have the same power [2, 3, 32], and we consider only the downlink scenario for simplicity because the description of such scenario in Section 2 involves all types of impairments that generate the noise. We consider MRT as precoding scheme adopted by the BS and perform the analysis under two scenarios: when a common local oscillator(CLO) is used at the BS and when separated oscillators(SLOs) are used. To this end, in subsection 3.1, based on the SINR, we derive closed-form expressions for the achievable data rate of a given user when CLO and SLOs are used, respectively. In subsection 3.2, closed-form expressions for the required downlink transmit power for desired user data rate are derived and presented.

\subsection{Achievable Data Rate under Hardware Impairments}

The closed-form expressions for the user data rate proposed in this paper follow the generally approximated one shown in literature as [20, 24, 26]

$$
R_{k}=\log _{2}\left(1+\operatorname{SINR}_{k}\right)
$$

Assuming equal power allocation [25, 26, 33], the SINR of $k^{\text {th }}$ user terminal is given as

$$
\operatorname{SINR}_{k}(t)=\frac{p\left|\mathbb{E}\left[\mathbf{h}_{k}^{H} \boldsymbol{\Theta}_{k}^{H}(t) \mathbf{f}_{k}\right]\right|^{2}}{\sum_{k=1}^{K} p \mathbb{E}\left[\left|\mathbf{h}_{k}^{H} \Theta_{k}^{H}(t) \mathbf{f}_{k}\right|^{2}\right]+\mathbb{E}\left[\left|\mathbf{h}_{k}^{H} \mathbf{\Theta}_{k}^{H}(t) \boldsymbol{\gamma}_{t}^{\mathrm{B}} \boldsymbol{\Theta}_{k}(t) \mathbf{h}_{k}\right|\right]+\mathbb{E}\left[v_{k, r}^{\mathrm{UT}}(t)\right]+\zeta^{\mathrm{DL}}}
$$


where $p$ is the power assigned to each user; $\left|\mathbb{E}\left[\mathbf{h}_{k}^{H} \Theta_{k}^{H}(t) \mathbf{f}_{k}\right]\right|^{2}$ reflects the gain of the desired signal at the user; $\mathbb{E}\left[\left|\mathbf{h}_{k}^{H} \Theta_{k}^{H}(t) \mathbf{f}_{k}\right|^{2}\right]$ reflects the multiuser interference caused by the signal intended for the $k^{\text {th }}$ user; $\mathbb{E}\left[\left|\mathbf{h}_{k}^{H} \boldsymbol{\Theta}_{k}^{H}(t) \boldsymbol{\gamma}_{t}^{\mathrm{BS}} \boldsymbol{\Theta}_{k}(t) \mathbf{h}_{k}\right|\right]$ originates from the HWIs at the BS, that is, the distortion noise $\eta_{t}^{\mathrm{BS}} ; \mathbb{E}\left[v_{k, r}^{\mathrm{UT}}(t)\right]$ originates from the HWIs at the $k^{\text {th }}$ user terminal, that is, the distortion noise $\eta_{r, k}^{\mathrm{UT}}$; and $\zeta^{\mathrm{DL}}$ is the downlink amplified thermal noise.

Unlike in [26] where the artificial noise is injected with the impact of uplink training, in this paper, we focus only on the channel estimation and downlink signal transmission without uplink training and artificial noise. This because the distortion noise generated at the base station is shown in [26] to have a similar effect as artificial noise. Following the guidelines in [26], we have

$$
\mathbb{E}\left[\mathbf{h}_{k}^{H} \Theta_{k}^{H}(t) \mathbf{f}_{k}\right]=\sqrt{\beta_{k} M} e^{-\frac{\sigma_{\psi}^{2}+\sigma_{\phi}^{2}}{2}}\left|t-t_{0}\right|
$$

The term $e^{-\frac{\sigma_{\psi}^{2}+\sigma_{\phi}^{2}}{2}\left|t-t_{0}\right|}$ reveals the effect of accumulated phase noise from the time $t_{0}$ the BS starts to estimate the channel to the time $t$ it starts to transmit signal. In other words, this interval of time corresponds to the one devoted for channel estimation, $\mu$ as illustrated in Fig. 2. Eq. (11) can then be rewritten as

$$
\mathbb{E}\left[\mathbf{h}_{k}^{H} \Theta_{k}^{H}(t) \mathbf{f}_{k}\right]=\sqrt{\beta_{k} M} e^{-\frac{\sigma_{\psi}^{2}+\sigma_{\phi}^{2}}{2} \mu}
$$

Moreover, we have

$$
\begin{gathered}
\mathbb{E}\left[\left|\mathbf{h}_{k}^{H} \boldsymbol{\Theta}_{k}^{H}(t) \mathbf{f}_{k}\right|^{2}\right]=\beta_{k}+\beta_{k}(M-1)\left(\frac{1-\epsilon+N_{0} \in}{N_{0}}\right) \\
\mathbb{E}\left[\left|\mathbf{h}_{k}^{H} \boldsymbol{\Theta}_{k}^{H}(t) \boldsymbol{\gamma}_{t}^{\mathrm{BS}} \boldsymbol{\Theta}_{k}(t) \mathbf{h}_{k}\right|\right]=\beta_{k} \kappa_{t}^{\mathrm{BS}} P_{d} \\
\mathbb{E}\left[v_{k, r}^{\mathrm{UT}}(t)\right]=\beta_{k} \kappa_{r}^{\mathrm{UT}} P_{d}
\end{gathered}
$$

where $P_{d}$ is the downlink transmit power and $\epsilon=e^{-\sigma_{\psi}^{2}}$.

For simplicity, we assume that the path-loss for all UT is identical, that is, $\beta_{k}=1$ [26]. Moreover, for equal power allocation, the power assigned to each user terminal is $p=$ $\frac{\phi P_{d}}{K}$, with $\phi \in(0,1]$ used for balance between data transmission and noise emission [26]. However, in this paper, we consider $\phi=1$ because the base station distortion noise is shown 
in [26] to be always beneficial when $\phi=1$.Therefore, substituting (12), (13), (14), (15), $\beta_{k}=1$ and $p=\frac{P_{d}}{K}$ into (10) gives

$$
\operatorname{SINR}_{k}(t)=\frac{P_{d} M e^{-\left(\sigma_{\psi}^{2}+\sigma_{\phi}^{2}\right) \mu}}{K\left[P_{d}\left(1+(M-1)\left(\frac{1-e^{-\sigma_{\psi}^{2}+N_{0} e^{-\sigma_{\psi}^{2}}}}{N_{0}}\right)+\kappa_{t}^{\mathrm{BS}}+\kappa_{r}^{\mathrm{UT}}\right)+\zeta^{\mathrm{DL}}\right]}
$$

Following the assumption widely made in the literature that the transmitter and the receiver hardware of each device are of the same quality $[19,20,22,25,26]$, we have the total downlink phase noise variance and distortion proportionality coefficient as $\sigma_{\psi}^{2}+\sigma_{\phi}^{2}=\sigma_{\theta}^{2}$ and $\kappa_{t}^{\mathrm{BS}}+\kappa_{r}^{\mathrm{UT}}=\kappa^{\mathrm{DL}}$, respectively. Therefore, the SINR of the $k^{\text {th }}$ user terminal is

$$
\operatorname{SINR}_{k}(t)=\frac{P_{d} M e^{-\sigma_{\theta}^{2} \mu}}{K\left[P_{d}\left(1+(M-1)\left(\frac{1-e^{-\sigma_{\psi}^{2}}+N_{0} e^{-\sigma_{\psi}^{2}}}{N_{0}}\right)+\kappa^{\mathrm{DL}}\right)+\zeta^{\mathrm{DL}}\right]}
$$

Next, we provide closed-form expressions for the $k^{\text {th }}$ user data rate with CLO and SLOs, respectively.

\subsubsection{User Data Rate with CLO}

In this configuration, the antenna arrays at the BS are co-located, and only one local oscillator is used and common to all the antennas, that is, $N_{0}=1$.This is known as synchronous operation at the base station, and the phase noise processes are identical for all $m=1, \ldots, M$ $[20,27]$. Substituting (17) and $N_{0}=1$ into (9), the achievable data rate of the $k^{\text {th }}$ user is then given as

$$
R_{k}^{\mathrm{CLO}}=\log _{2}\left[1+\frac{P_{d} M e^{-\sigma_{\theta}^{2} \mu}}{K\left[P_{d}\left(M+\kappa^{\mathrm{DL}}\right)+\zeta^{\mathrm{DL}}\right]}\right]
$$

\subsubsection{User Data Rate with SLOs}

In this configuration, the antenna arrays at the BS are distributed, and each antenna uses a separate local oscillator, that is, $N_{0}=M$.This is known as non-synchronous operation at the base station, and the base station antennas have independent phase noise processes for all $m=1, \ldots, M[20,27]$. However, for simplicity, we assume i.i.d. phase noise statistics across different antennas and user terminals, that is, $\sigma_{\psi}^{2}=\sigma_{\phi}^{2}, \forall m, k[20,27]$. Substituting (17) and $N_{0}=M$ into (9), the achievable data rate of the $k^{\text {th }}$ user is then given as 


$$
R_{k}^{\mathrm{SLOs}}=\log _{2}\left[1+\frac{P_{d} M e^{-\sigma_{\theta}^{2} \mu}}{K\left(P_{d}\left(1+(M-1)\left(\frac{1-e^{-\sigma_{\psi}^{2}}+M e^{-\sigma_{\psi}^{2}}}{M}\right)+\kappa^{\mathrm{DL}}\right)+\zeta^{\mathrm{DL}}\right)}\right]
$$

Considering and analyzing Eq. (9), (17), (18) and (19) it is clear that the various hardware impairments have an impact on the user data rates. Especially, given that the additive distortion noise and amplified thermal noise parameters are found in the denominator of $R_{k}^{\mathrm{CLO}}$ and $R_{k}^{\mathrm{SLOs}}$ expressions, their presence decreases the rates. Moreover, the presence of the phase noise parameters in both the numerator and denominator of the expressions also impacts the rates. Furthermore, the number of local oscillators which is related to $M$ and the time devoted to channel estimation also affect the user data rates. More insight about the impact of the HWIs and the number of local oscillators is provided by simulation results in Section 5 .

\subsection{Required Downlink Transmit Power}

Here, it is supposed that the downlink transmit power is not fixed, and we derive its closed-form expressions when CLO and SLOs are used, respectively.

Let's rewrite the user data rate $R_{k}^{\mathrm{CLO}}$ as

$$
R_{k}=\log _{2}\left[1+\frac{P_{d} M e^{-\sigma_{\theta}^{2} \mu}}{K\left[P_{d}\left(M+\kappa^{\mathrm{DL}}\right)+\zeta^{\mathrm{DL}}\right]}\right]
$$

Given that the desired user data rate is positive, we have

$$
1+\frac{P_{d} M e^{-\sigma_{\theta}^{2} \mu}}{K\left[P_{d}\left(M+\kappa^{\mathrm{DL}}\right)+\zeta^{\mathrm{DL}}\right]}>0
$$

We can then apply the property of logarithm function which states that $\log _{b}(a)=\frac{\log (a)}{\log (b)}$

If $a>0$. Applying such property to (21) we get

$$
R_{k}=\frac{\ln \left[1+\frac{P_{d} M e^{-\sigma_{\theta}^{2} \mu}}{K\left[P_{d}\left(M+\kappa^{\mathrm{DL}}\right)+\zeta^{\mathrm{DL}}\right]}\right]}{\ln 2}
$$

It gives

$$
(\ln 2) R_{k}=\ln \left[1+\frac{P_{d} M e^{-\sigma_{\theta}^{2} \mu}}{K\left[P_{d}\left(M+\kappa^{\mathrm{DL}}\right)+\zeta^{\mathrm{DL}}\right]}\right]
$$

Applyimg exponential to both sides gives

$$
1+\frac{P_{d} M e^{-\sigma_{\theta}^{2} \mu}}{K\left[P_{d}\left(M+\kappa^{\mathrm{DL}}\right)+\zeta^{\mathrm{DL}}\right]}=e^{(\ln 2) R_{k}}
$$


Rearranging (24) and applying cross product give

$$
P_{d} M e^{-\sigma_{\theta}^{2} \mu}=\left(e^{(\ln 2) R_{k}}-1\right)\left(K P_{d} M+K P_{d} \kappa^{\mathrm{DL}}+K \zeta^{\mathrm{DL}}\right)
$$

Making $P_{d}$ the subject and further derivations give the required downlink transmit power when common local oscillator is used as

$$
P_{d}^{\mathrm{CLO}}=\frac{K \zeta^{\mathrm{DL}}\left(e^{(\ln 2) R_{k}}-1\right)}{M\left(e^{-\sigma_{\theta}^{2} \mu}-K e^{(\ln 2) R_{k}}+K\right)+K \kappa^{\mathrm{DL}}\left(1-e^{(\ln 2) R_{k}}\right)}
$$

Similar to CLO case, we derive the required downlink transmit power when separate local oscillators are used as

$$
P_{d}^{\mathrm{SLOS}}=\frac{K \zeta^{\mathrm{DL}}\left(e^{(\ln 2) R_{k}}-1\right)}{M e^{-\sigma_{\theta}^{2} \mu}-K A\left(e^{(\ln 2) R_{k}}-1\right)}
$$

where

$$
A=2-2 e^{-\sigma_{\psi}^{2}}+M e^{-\sigma_{\psi}^{2}}-\frac{1}{M}+\frac{e^{-\sigma_{\psi}^{2}}}{M}+\kappa^{\mathrm{DL}}
$$

The analysis is similar to the one under user data rate, and more insight about the impact of HWIs and the number of local oscillators on the required downlink transmit power is also provided in Section 5.

\section{Proposed Approach to Minimize the Effect of Hardware Impairments: Cell Size Reduction}

As mentioned earlier in subsection 3.1, the term $e^{-\frac{\sigma_{\psi}^{2}+\sigma_{\phi}^{2}}{2}\left|t-t_{0}\right|}$ reveals the effect of accumulated phase noise during channel estimation time, , and its squared form appears in Eq. (18) and (19) as $e^{-\sigma_{\theta}^{2} \mu}$. Moreover, it is shown in [34] that the fraction of time devoted to channel estimation is related to the cell radius $R$ as

$$
3 R^{2}=\frac{\mu T_{t}}{\pi \rho}
$$

where $\rho$ is the co-channel cells user density. Then we get

$$
\mu=\frac{3 \pi \rho R^{2}}{T_{t}}
$$

Substituting (30) into (18) and (19) gives the user data rate for CLO and SLOs configurations as a function of cell radius as 


$$
R_{k}^{\mathrm{CLO}}=\log _{2}\left[1+\frac{P_{d} M e^{-\frac{3 \pi \rho \sigma_{\theta}^{2} R^{2}}{T_{t}}}}{K\left[P_{d}\left(M+\kappa^{\mathrm{DL}}\right)+\zeta^{\mathrm{DL}}\right]}\right]
$$

and

$$
R_{k}^{\mathrm{SLOS}}=\log _{2}\left[1+\frac{P_{d} M e^{-\frac{3 \pi \rho \sigma_{\theta}^{2} R^{2}}{T_{t}}}}{K\left(P_{d}\left(1+(M-1)\left(\frac{1-e^{-\sigma_{\psi}^{2}}+M e^{-\sigma_{\psi}^{2}}}{M}\right)+\kappa^{\mathrm{DL}}\right)+\zeta^{\mathrm{DL}}\right)}\right]
$$

respectively.

We also substitute (30) into (26) and (27) to get the required downlink transmit power for CLO and SLOs configurations as a function of cell radius as

and

$$
P_{d}^{\mathrm{CLO}}=\frac{K \zeta^{\mathrm{DL}}\left(e^{\left.(\ln 2) R_{k}-1\right)}\right.}{M\left(e^{-\frac{3 \pi \rho \sigma_{\theta}^{2} R^{2}}{T} t}-K e^{(\ln 2) R_{k}}+K\right)+K \kappa^{\mathrm{DL}}\left(1-e^{(\ln 2) R_{k}}\right)}
$$

$$
P_{d}^{\mathrm{SLOs}}=\frac{K \zeta^{\mathrm{DL}}\left(e^{\left.(\ln 2) R_{k-1}\right)}\right.}{M e^{-\frac{3 \pi \rho \sigma_{\theta}^{2} R^{2}}{T_{t}}}-K A\left(e^{\left.(\ln 2) R_{k}-1\right)}\right.}
$$

respectively. $A$ is the same as (28).

Our main goal is to minimize the effect of accumulated phase noise during channel estimation time in order to increase the user capacity and decrease the transmit power.

Considering Eq. (31) and (32), for other parameters given, reducing $R$ will increase the value of $e^{-\frac{3 \pi \rho \sigma_{\theta}^{2} R^{2}}{T_{t}}}$ and hence the values of $R_{k}^{C L O}$ and $R_{k}^{S L O S}$ will increase.

Similarly, considering Eq. (33) and (34), when the value of $e^{-\frac{3 \pi \rho \sigma_{\theta}^{2} R^{2}}{T_{t}}}$ increases with a reduction of $R$, the values of $P_{d}^{C L O}$ and $P_{d}^{S L O S}$ will decrease because their denominators will increase.

From the analysis, we then conclude that reducing the cell radius can achieve our main goal.

\section{Simulation Results}

In this section, analytical and simulation results are provided to evaluate the theoretical analysis presented in Section 3 and Section 4. Simulations are performed over 5000 channel realizations using Monte Carlo. Without loss of generality, some simulation parameters from $[19,20,22,23,25,26,33,34]$ are used throughout our simulations. We consider one cell whose radius is initially fixed at $2000 \mathrm{~m}$. The system bandwidth is regarded as $20 \mathrm{MHz}$, with a carrier frequency equal to $2 \mathrm{GHz}$. It is assumed that the base station transmits data to all the users with the same power [25, 26, 33], and the average system SNR is $15 \mathrm{~dB}$. We use $0.06^{\circ}$ as typical values for the phase noise increment standard deviations, $\sigma_{\psi}$ and $\sigma_{\phi}$, adopted in the 
long-term evolution (LTE) specifications [35]. Furthermore, we use $0.05^{2}$ as typical values for the additive distortion noise proportionality coefficients $\kappa_{t}^{\mathrm{BS}}$ and $\kappa_{r}^{\mathrm{UT}}$ [19], and the downlink amplified receiver noise is set to $\zeta^{\mathrm{DL}}=1.58$ [20]. As shown in the figures, simulation and analytical results almost coincide in all the cases.

\subsection{Achievable User Data Rate}

In order to evaluate the impact of the transceiver hardware impairments on the performance of the downlink system, we first plotted the achievable user data rate against the number of base station antennas for CLO and SLOs configurations and for the case where it is assumed that there is no HWIs or other performance limiting factors and the CSI is perfect. To this end, we used Eq. (18) and (19) for CLO and SLOs, respectively, and for no HWIs case, we used the following Eq. [36] $: R_{k}=\log _{2}\left[1+\left(P_{d} M / K\left(P_{d}+1\right)\right)\right]$.The downlink transmit power is fixed at $43 \mathrm{dBm}$; the fraction of time devoted to channel estimation is initially fixed at $0.3 \mathrm{~ms}$ [34], and the simulations are performed for 10 and 20 users for all the three cases. The results are depicted in Fig. 3 which then provides a comparison of the user data rate versus the number of BS antennas in cases of HWIs and no HWIs, and also a comparison between CLO and SLOs configurations under HWIs. The results show that despite the HWIs, the user data rate increases as the number of BS antennas increases. However, compared to the ideal case where there are no hardware impairments, there is a performance loss at every $M$, but the curves still increase with $M$. These results, therefore, confirm that HWIs produce a divergence between the transmitted signal and what is really generated. It distorts the received signal in the detecting process and then limits the accuracy of the channel estimation and hence the capacity of every user.

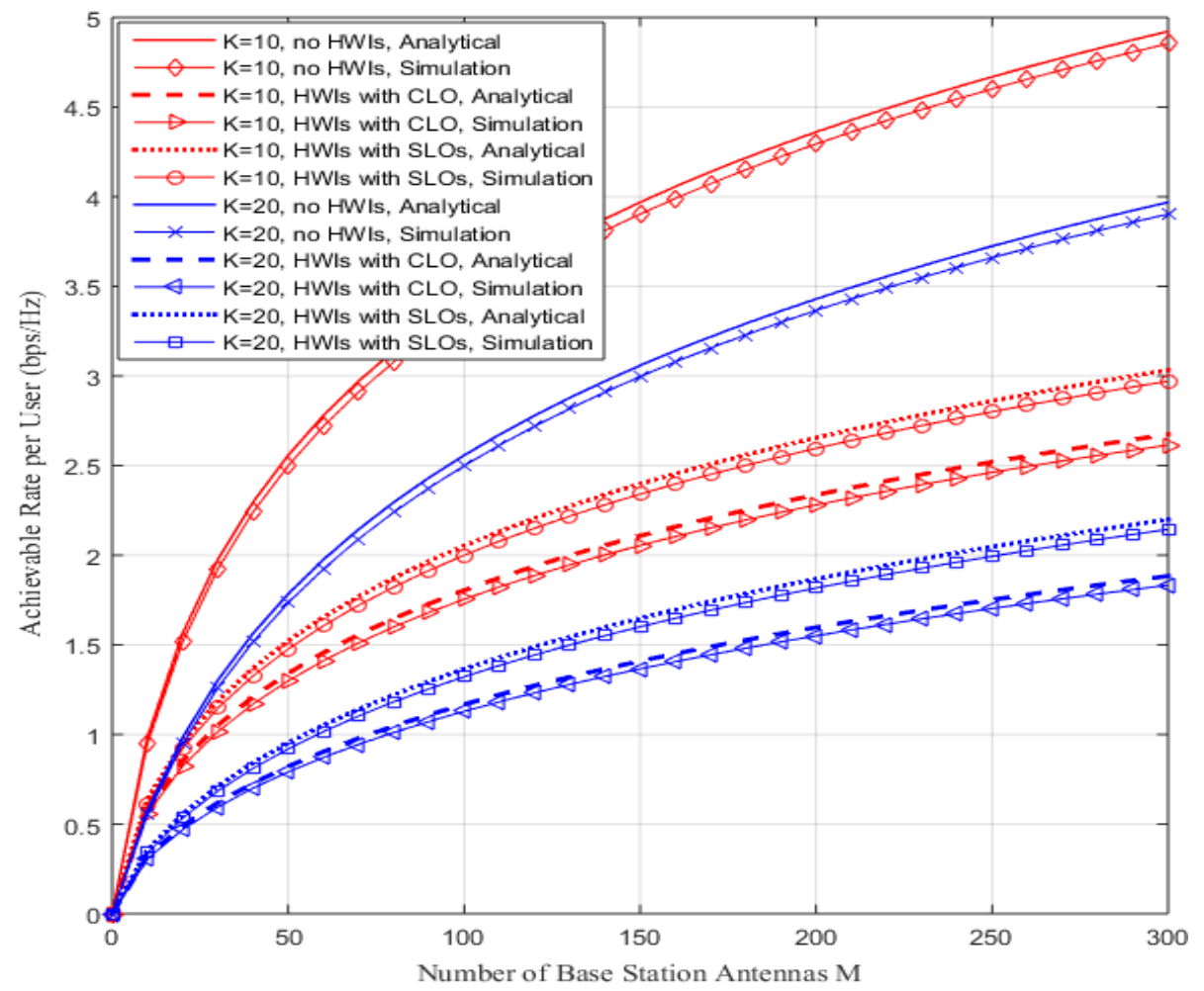

Fig. 3. User data rate versus number of base station antennas with $K=\{10,20\}$ and $P_{d}=43 \mathrm{dBm}$ 
Moreover, a comparison of the performance of CLO and SLOs shows a smaller rate loss for SLOs. This also confirms that large- $M$ behavior depends strongly on the oscillators. SLOs provides larger user data rate than CLO at the expensive of increasing the deployment cost by having $M$ local oscillators. Furthermore, the results show that the user data rate decreases at a higher number of users. This can be justified by the fact that as the number of BS antennas grows large, the detrimental effect of HWIs at the BS vanishes almost completely because the noises are spread in arbitrary directions, and the spatial resolution of the array increases and enables exact transmit beamforming for the useful signal. In contrast, the noises at the users' side do not vanish and behave as interferers with the same effective channels as the useful signal. Hence, only the hardware impairments at the users mainly limit the downlink capacity.

\subsection{Required Downlink Transmit Power}

In the following figures, only HWIs cases are investigated. The metric under investigation is the downlink transmit power required for some desired user data rate under CLO and SLOs scenarios. We then used Eq. (26) and (27) in the simulation to plot the required downlink transmit power against the desired user rate.

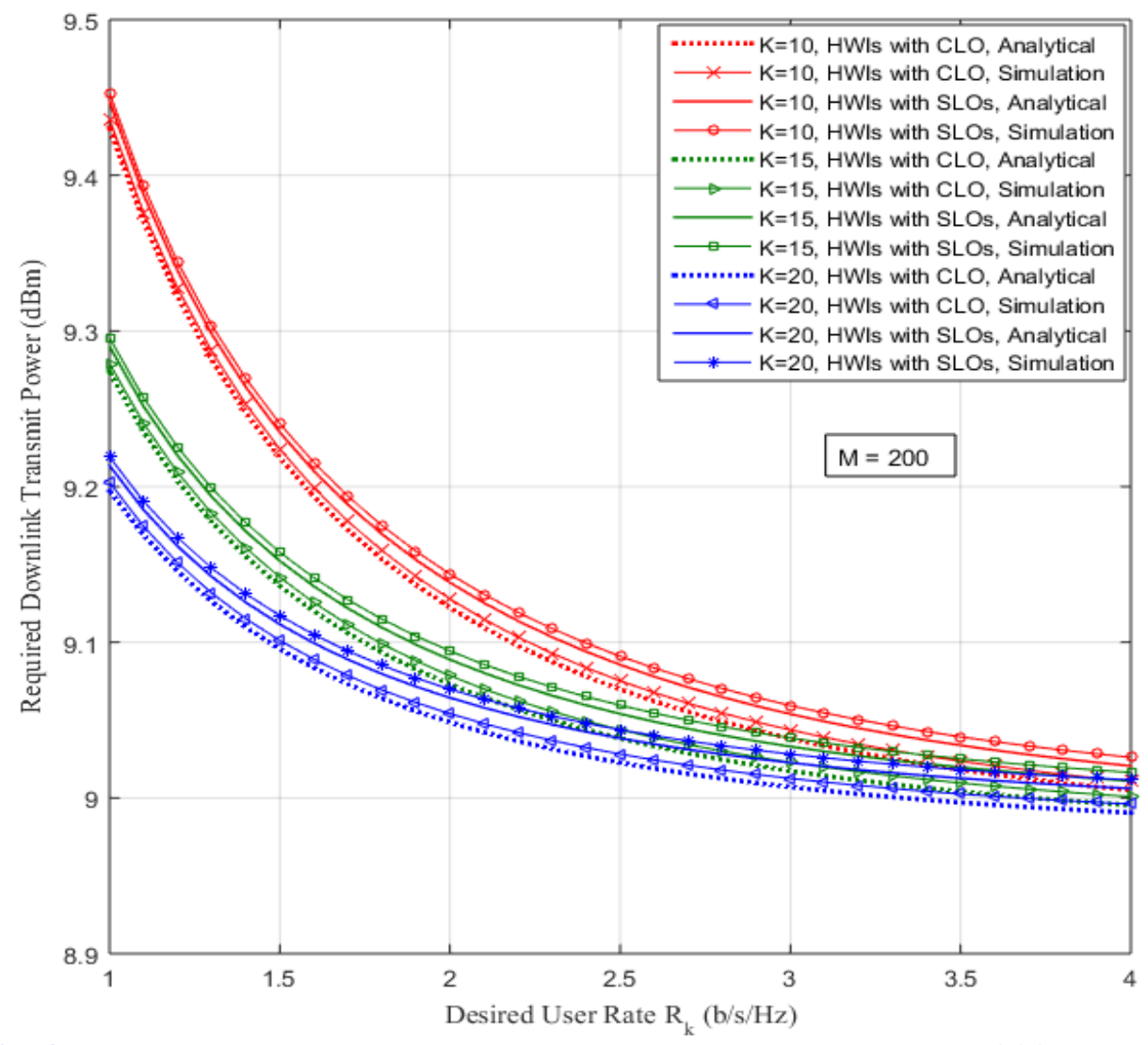

Fig. 4. Required downlink transmit power versus desired user rate with $\boldsymbol{M}=\mathbf{2 0 0}$ and $\boldsymbol{K}=$

$\{10,15,20\}$ 


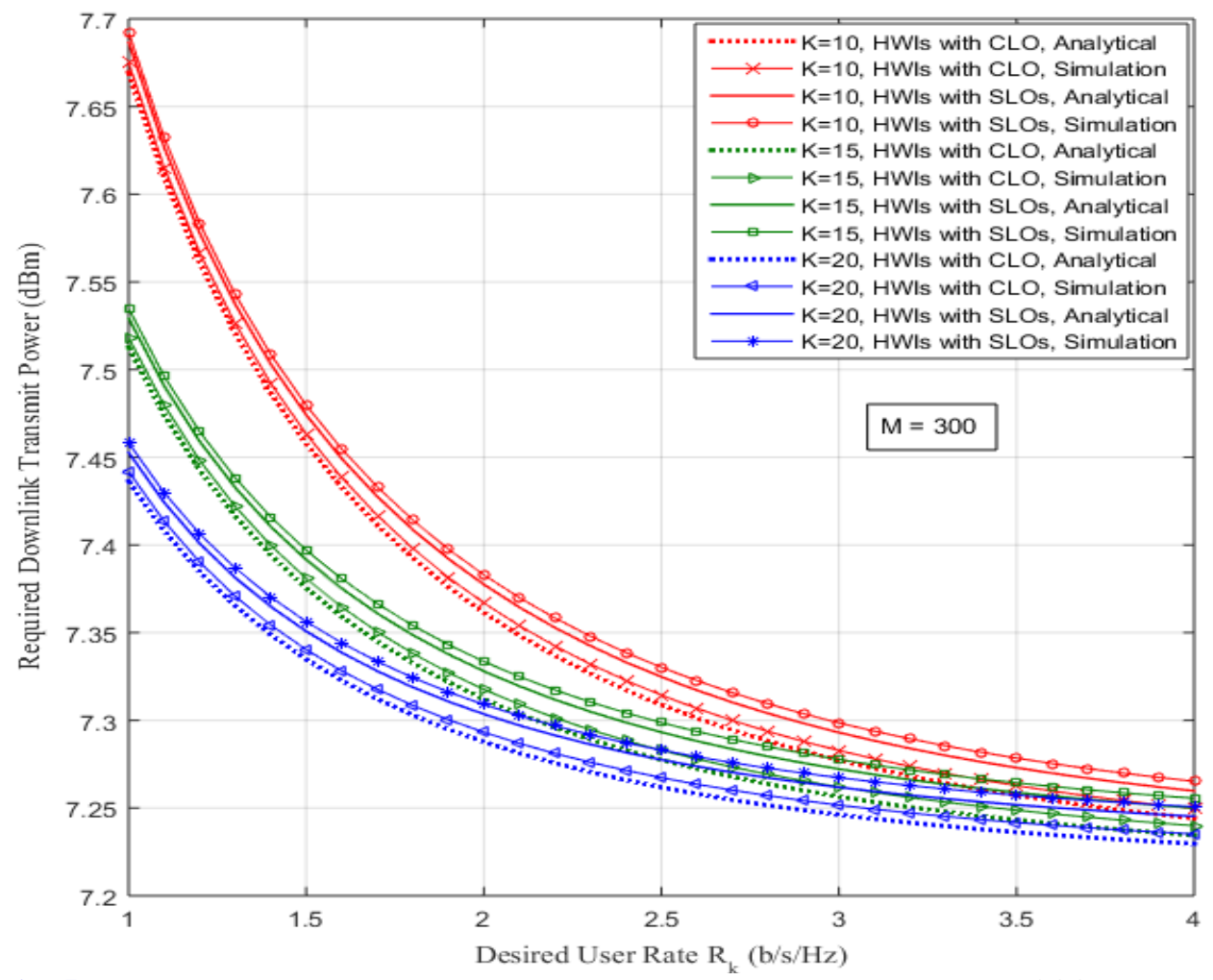

Fig. 5. Required downlink transmit power versus desired user rate with $\boldsymbol{M}=\mathbf{3 0 0}$ and $\boldsymbol{K}=$

$\{10,15,20\}$

In Fig. 4 and Fig. 5 the number of base station antennas is fixed at 200 and 300, respectively, and the simulations are performed for 10; 15 and 20 users for both CLO and SLOs configurations. The results show that the power decreases as the desired user rate increases; which is in agreement with one of massive MIMO technology advantages aiming at getting higher data rate at lower power consumption. Moreover, the results show more power consumption with SLOs than with CLO. The reason for this is that more local oscillators are used under SLOs configuration and the more the number of local oscillators the higher the power consumed at the base station. Furthermore, in both configurations, the power consumption is less at a higher number of users. This can be justified by the fact that when the channel state information is imperfect, there is a gain in the required power consumption for per-user rate larger than a certain treshold as the number of users increases [37]. Also, a comparison of the results in both figures shows that the higher the number of base station antennas deployed, the lower the required power consumption, which is also in agreement with the principle of massive MIMO technology stating that at higher number of antennas at the transmit or receive side, the energy can be focused with extreme sharpness into small regions, which results in an improvement of the data rates and reliability and a reduction of the required transmit power [1-4], [24].

In Fig. 6 and Fig. 7 the number of users is fixed at 10 and 20, respectively, and the simulations are performed for 200; 250 and 300 base station antennas for both CLO and SLOs configurations. The results are similar to the ones in Fig. 4 and Fig. 5. That is, the power decreases as the desired user rate increases; more power is consumed with SLOs than with CLO; less power is consumed at a higher number of base station antennas; the higher the number of users, the lower the required power consumption. 


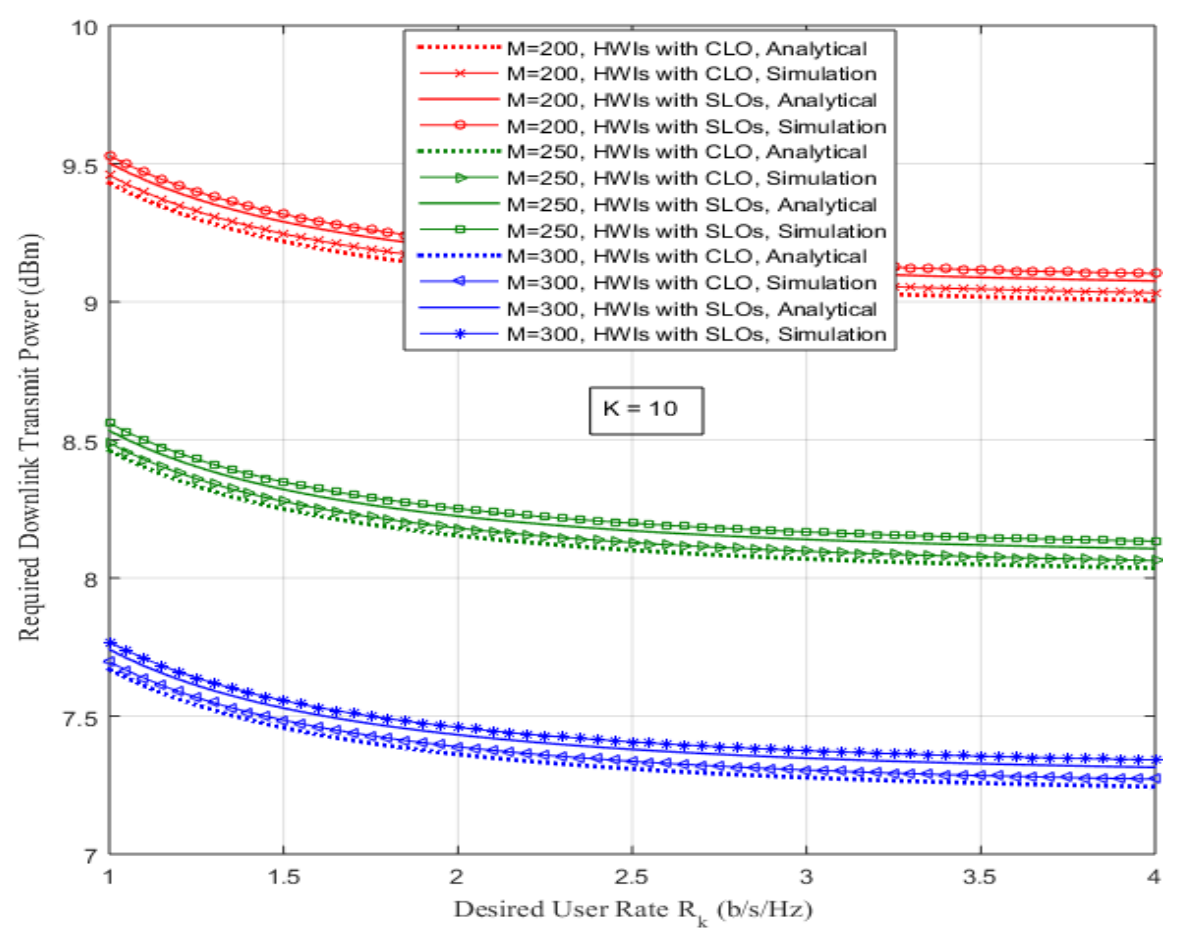

Fig. 6. Required downlink transmit power versus desired user rate with $\boldsymbol{K}=\mathbf{1 0}$ and $M=\{200,250,300\}$

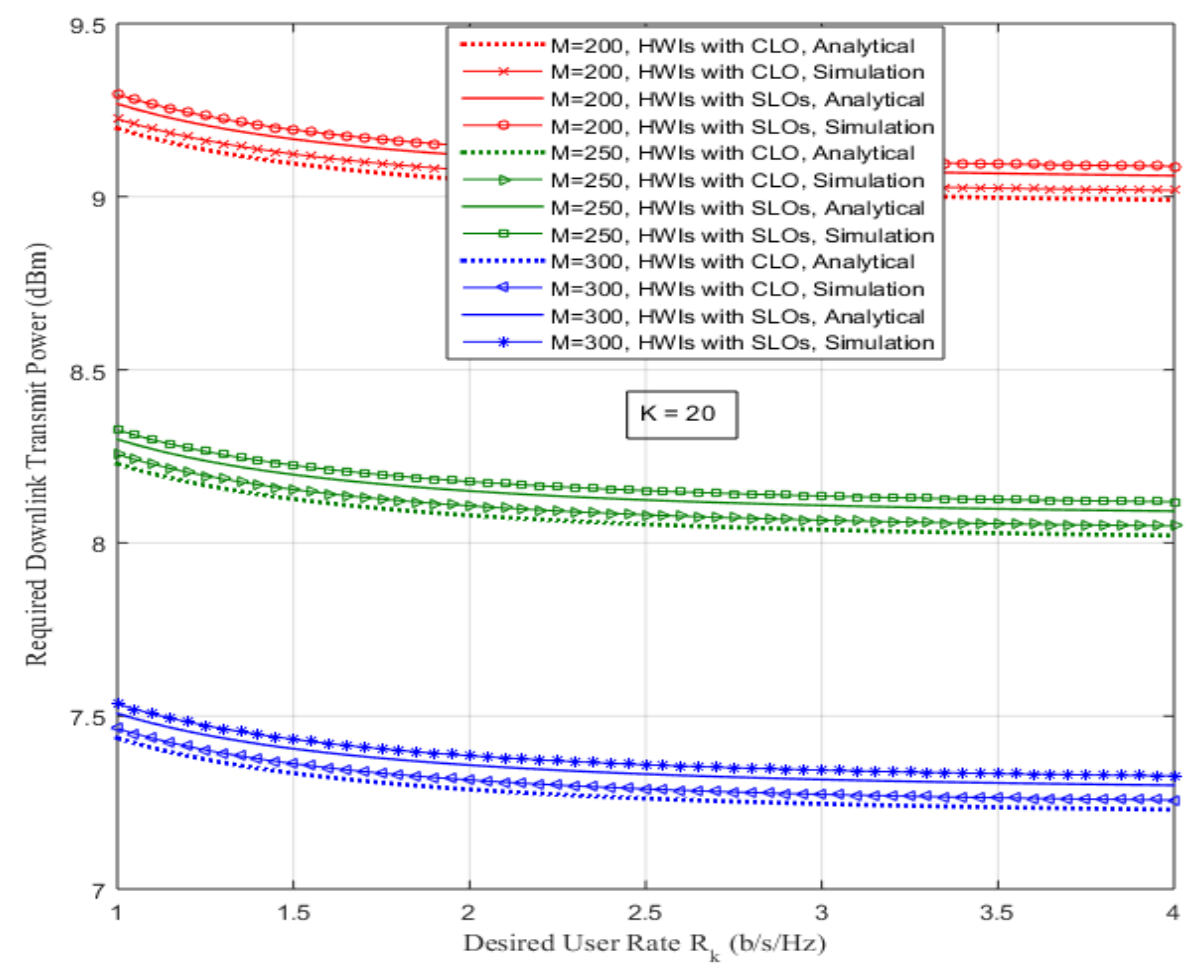

Fig. 7. Required downlink transmit power versus desired user rate with $\boldsymbol{K}=\mathbf{2 0}$ and $M=\{200,250,300\}$ 


\subsection{Effect of Cell Size Reduction}

In this subsection, we investigate on the effect of the cell radius reduction on the HWIs and hence on the user data rate, and required transmit power. To this end, we consider Eq. (31) and (32) in the simulation to plot the user data rate against the cell radius for CLO and SLOs configurations, respectively, and Eq. (33) and (34) to plot the required downlink transmit power against the cell radius for CLO and SLOs, respectively. The results are depicted in Fig. 8 and Fig. 9 for the user data rate, and in Fig. 10 and Fig. 11 for the required downlink transmit power. The cell radius is varied from $20 \mathrm{~m}$ to $2000 \mathrm{~m}$, and the uplink training length is $10^{6}$ time slots [34]. The co-channel cells user density is set to 0.005 users per square meter, and this parameter is shown in [34] not to vary as the radius of the cell of interest is reduced. This is because it is the user density in the area whose radius is the co-channel cell distance, and that distance does not vary as we reduce the radius of the cell of interest. The user data rate is set at 2 b/s/Hz for Fig. 10 and Fig. 11.

In Fig. 8, the number of BS antennas is fixed at 300, and the simulations are performed for 10; 15 and 20 users for both CLO and SLOs configurations. The results show that as the cell radius is reduced, the user data rate significantly increases despite the presence of HWIs. Moreover, the results show lower data rate for higher number of users for both configurations, which is in agreement with the results from Fig. 3.

In Fig. 9, the number of users is fixed at 20, and the simulations are performed for 200; 250 and 300 BS antennas for both configurations. Similar to Fig. 8, the results show a significant increase of the user data rate as the cell radius is reduced. Also, it is shown for both configurations that the higher the number of BS antennas the higher the user data rate, which is also in agreement with the results from Fig. 3.

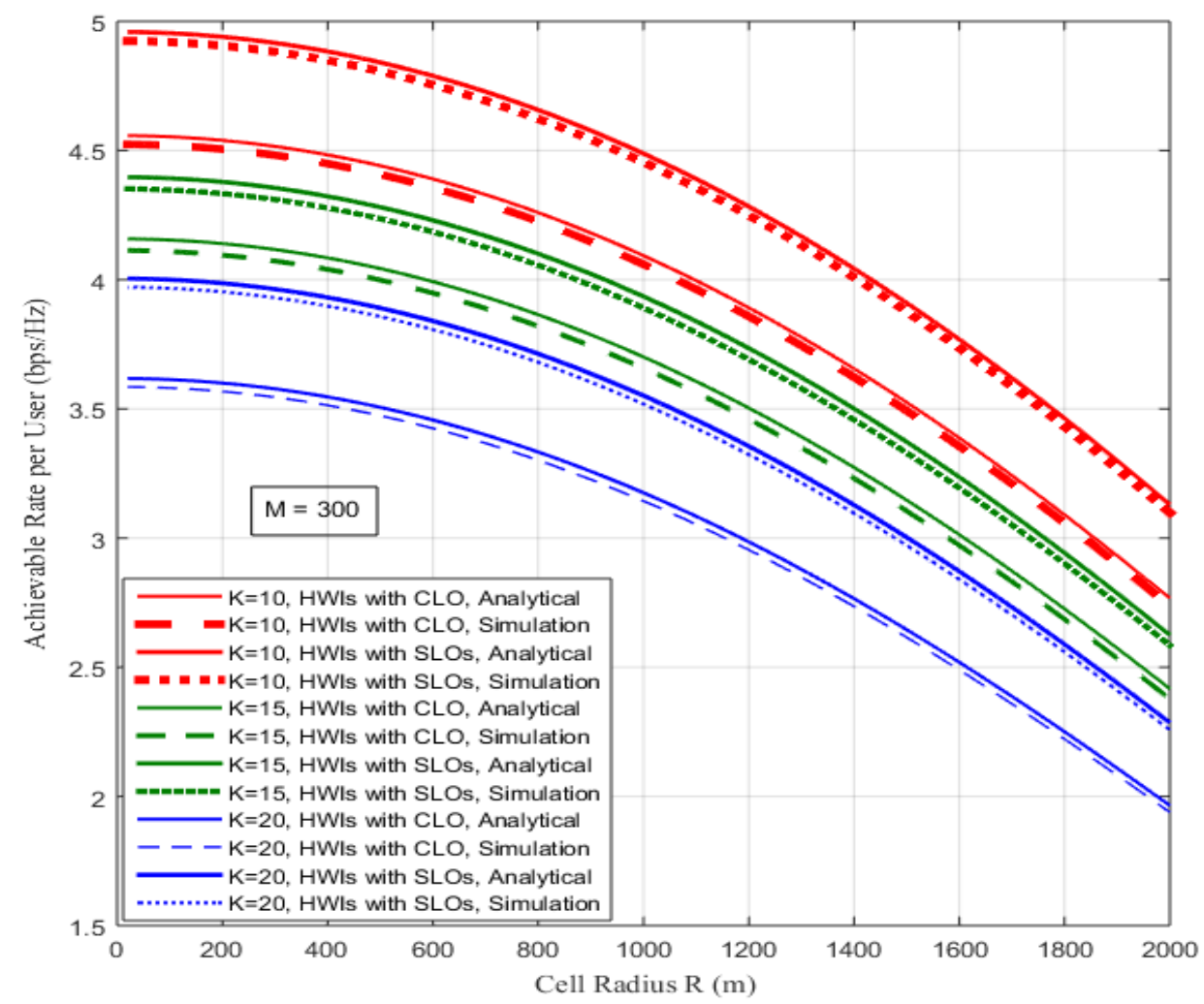

Fig. 8. User data rate versus cell radius with $\boldsymbol{M}=\mathbf{3 0 0}$ and $\boldsymbol{K}=\{\mathbf{1 0}, \mathbf{1 5}, \mathbf{2 0}\}$ 


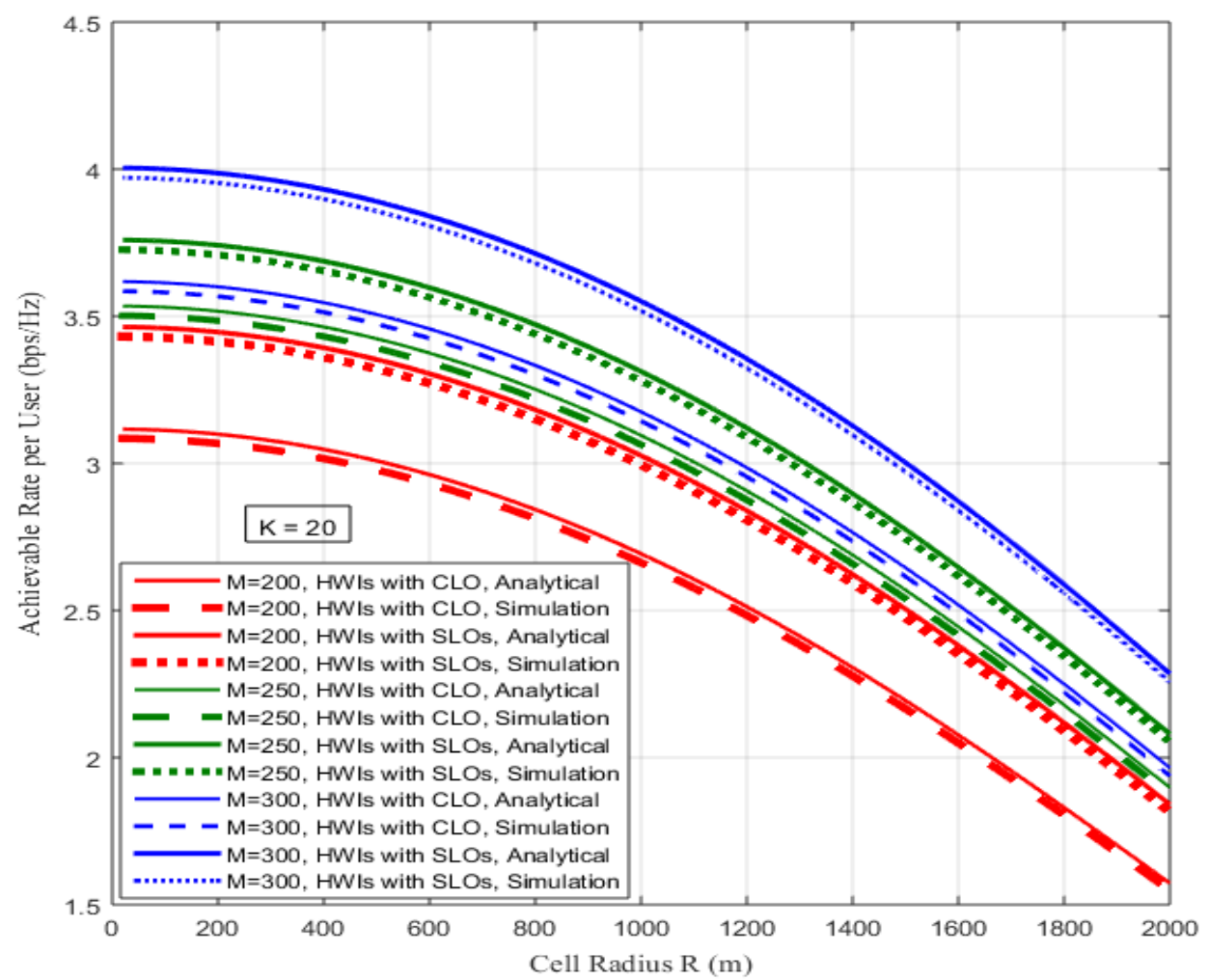

Fig. 9. User data rate versus cell radius with $K=\mathbf{2 0}$ and $\boldsymbol{M}=\{\mathbf{2 0 0}, \mathbf{2 5 0}, \mathbf{3 0 0}\}$

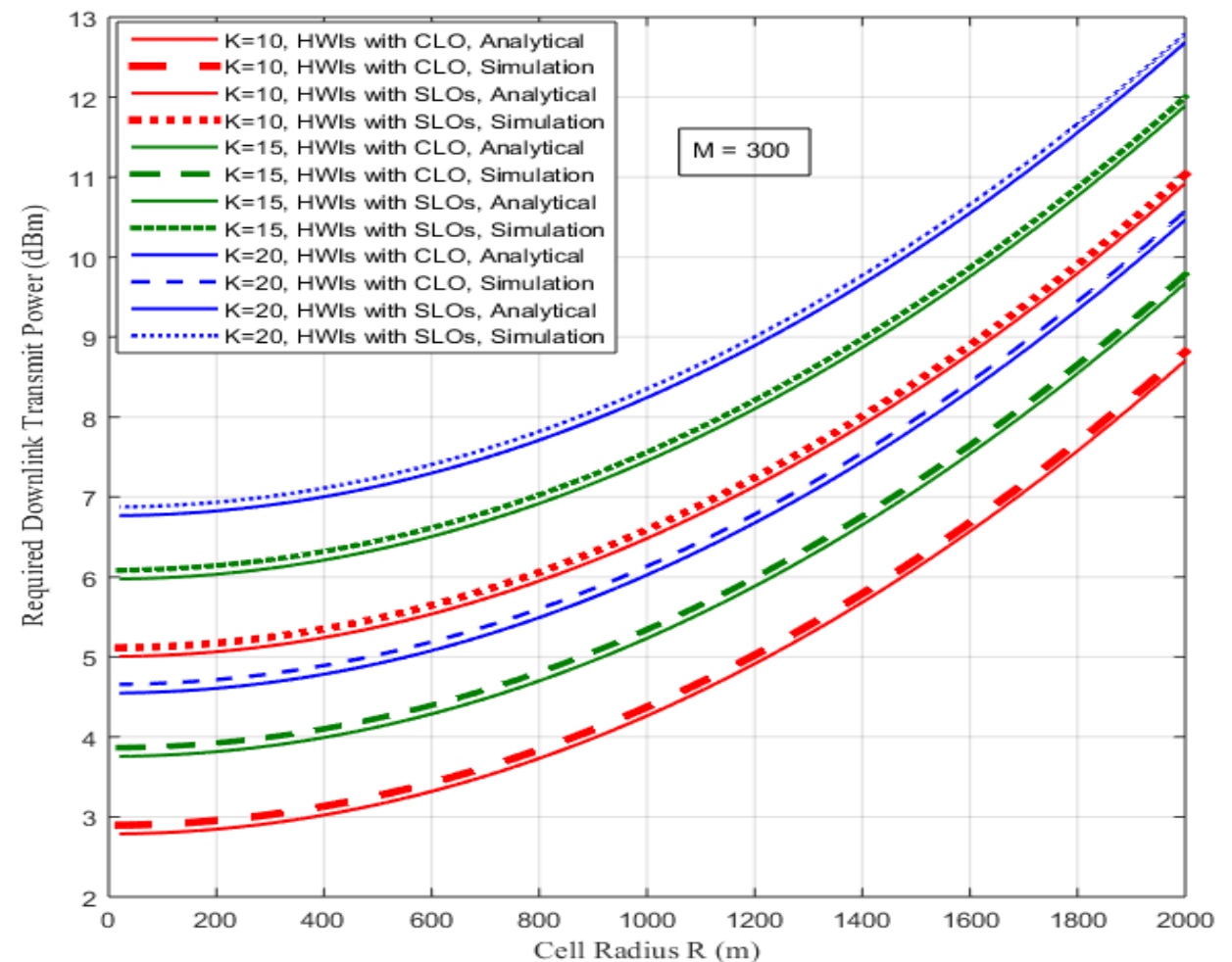

Fig. 10. Required downlink transmit power versus cell radius with $\boldsymbol{M}=\mathbf{3 0 0}$ and $\boldsymbol{K}=\{\mathbf{1 0}, \mathbf{1 5}, \mathbf{2 0}\}$ 


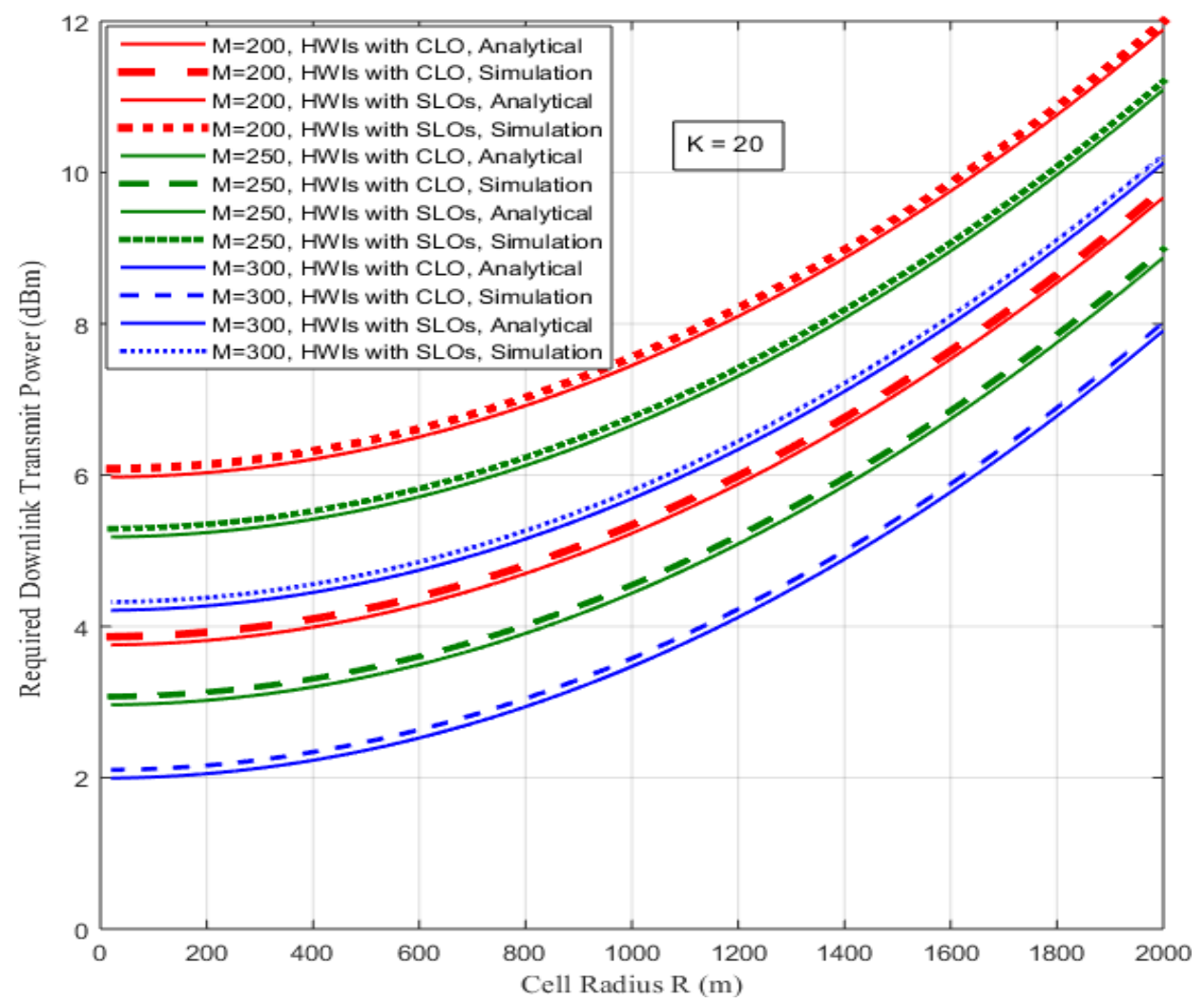

Fig. 11. Required downlink transmit power versus cell radius with $K=\mathbf{2 0}$ and $\boldsymbol{M}=\{\mathbf{2 0 0}, \mathbf{2 5 0}, \mathbf{3 0 0}\}$

Fig. 10 depicts the results for the number of BS antennas fixed at 300, and the simulations performed for 10; 15 and 20 users for both CLO and SLOs configurations. The results show a significant decrease of the total downlink transmit power as the cell radius is reduced. Moreover, the results show higher power for higher number of users for both configurations. This is because the per-user rate and the number of BS antennas are fixed.

In Fig. 11, the number of users is fixed at 20, and the simulations are performed for 200; 250 and 300 BS antennas for both configurations. Similar to Fig. 10, the total downlink transmit power significantly decreases as the cell radius is reduced. It is also shown for both configurations that the higher the number of BS antennas the lower the required power consumption, which is in agreement with the results from Fig. 6 and Fig. 7.

From the results obtained from Fig. 8, Fig. 9, Fig. 10 and Fig. 11. we can conclude that cell size reduction can minimize the effect of HWIs in massive MIMO system and hence improve the user data rate, and reduce the transmit power. More explicitly, it can minimize the effect of accumulated phase noise during channel estimation time which has an impact on the downlink performance.

A performance comparison between the results from our proposed approach and some existing referenced results is shown Table $\mathbf{1}$. We consider the data rate for the comparison and the values are chosen for the cell radius reduced to $50 \mathrm{~m}$ (because our approach is based on cell size reduction). It is realized that our approach offers a competitive advantage than most of the referenced results in terms of user data rate. 
Table 1. Results comparison

\begin{tabular}{|c|c|c|c|c|c|}
\hline References & $\begin{array}{l}\text { Carrier } \\
\text { frequency }\end{array}$ & System SNR & $\begin{array}{l}\text { Number of } \\
\text { BS antennas }\end{array}$ & $\begin{array}{l}\text { Number } \\
\text { of users }\end{array}$ & $\begin{array}{c}\text { Spectral efficiency(SE) } \\
\text { or User data rate } \boldsymbol{R}_{\boldsymbol{k}}\end{array}$ \\
\hline [19] & $2 \mathrm{GHz}$ & $20 \mathrm{~dB}$ & 1000 & 8 & $\mathrm{SE} \approx 3.5$ bits/channel use \\
\hline [20] & $2 \mathrm{GHz}$ & $15 \mathrm{~dB}$ & 400 & 8 & $\begin{array}{c}\boldsymbol{R}_{\boldsymbol{k}} \approx 3 \text { bits/channel use for } \\
\text { CLO } \\
\boldsymbol{R}_{\boldsymbol{k}} \approx 3.5 \text { bits/channel use } \\
\text { for SLOs }\end{array}$ \\
\hline$[21]$ & $2 \mathrm{GHz}$ & $20 \mathrm{~dB}$ & 50 & 10 & $\boldsymbol{R}_{\boldsymbol{k}} \approx 1.5$ bits/channel use \\
\hline$[24]$ & $2 \mathrm{GHz}$ & $35 \mathrm{~dB}$ & 500 & 5 & $\boldsymbol{R}_{\boldsymbol{k}} \approx 10 \mathrm{~b} / \mathrm{s} / \mathrm{Hz}$ \\
\hline This work & $2 \mathrm{GHz}$ & $15 \mathrm{~dB}$ & 300 & 10 & $\begin{array}{l}\boldsymbol{R}_{\boldsymbol{k}} \approx 4.4 \mathrm{~b} / \mathrm{s} / \mathrm{Hz} \text { for CLO } \\
\boldsymbol{R}_{\boldsymbol{k}} \approx 4.9 \mathrm{~b} / \mathrm{s} / \mathrm{Hz} \text { for SLOs }\end{array}$ \\
\hline
\end{tabular}

\section{Conclusions}

In this paper, we analyzed the performance of a single-cell downlink system in the presence of transceiver hardware impairments, using maximum ratio transmission (MRT) as precoding scheme. Our analysis involves two scenarios: synchronous operation at the base station where only one local oscillator is used and common to all the antennas (CLO), and non-synchronous operation at the base station where each antenna uses a separate local oscillator (SLO). We derived closed-form expressions for a typical user data rate under each scenario, and showed that, though the rate increases with the number of base station antennas, there is still some loss due to the transceiver hardware impairments. Moreover, we showed that higher data rate could be achieved when SLOs are used; and the rate decreases at a higher number of users under both scenarios. We then conclude that as the number of base station antennas grows large, it is only the hardware impairments at the users that mainly limit the downlink capacity. Furthermore, we derived closed-form expressions for the downlink transmit power required for some desired per-user data rate under each scenario. Our results show that as the desired per-user rate increases, the transmit power decreases and the power is higher under SLOs than under CLO. The results also show under both scenarios that, the higher the fixed value of the number of base station antennas or users, the lower the required power. We, therefore, conclude that massive MIMO downlink system with transceiver hardware impairments experiences lower data rate loss with separate local oscillators used at the base station, and the lower rate loss is at the price of higher power consumption compared to the use of a common local oscillator. Finally, we proposed an approach to minimize the effect of HWIs on the system performance. Our approach consists of reducing the cell size, and the results show that when the cell size is reduced the effect of accumulated phase noise during channel estimation time is minimized and hence the user capacity is increased, and the downlink transmit power is decreased. Even though our approach will result in deploying more cell sites in the network, it meets the advantages of small cells deployment which is also an emerging technique for the next generation cellular systems. Such advantages include: increasing capacity in areas with higher user densities; improving the network energy efficiency; extending handset battery life by reduced power consumption. 


\section{References}

[1] E. G. Larson, O. Edfors, F. Tufvesson, and T. L. Marzetta, "Massive MIMO for Next Generation Wireless Systems,” IEEE Communications Magazine, vol. 52, no. 2, pp. 186-195, February, 2014. Article (CrossRef Link).

[2] F. Rusek, D. Persson, B. K. Lau, E. G. Larsson, T. L. Marzetta, O. Edfors, and F. Tufvesson, "Scaling up MIMO : Opportunities and Challenges With Very Large Arrays," IEEE Signal Processing Magazine, vol. 30, no. 1, pp. 40-60, January, 2013. Article (CrossRef Link).

[3] H. Q. Ngo, E. G. Larsson, and T. L. Marzetta, "Energy and Spectral Efficiency of Very Large Multiuser MIMO Systems,” IEEE Transactions on Communications, vol. 61, no. 4, pp. 1436-1449, April, 2013. Article (CrossRef Link).

[4] J. Kang, J. Kang, N. Lee, B. M. Lee, and J. Bang, "Minimizing Transmit Power for Cooperative Multicell System with Massive MIMO," in Proc. of IEEE 10th Consumer Communications and Networking Conference, (CCNC), pp. 438-442, January 10-13, 2013. Article (CrossRef Link).

[5] J. Hoydis, S. Ten Brink, and M. Debbah, "Comparison of linear precoding schemes for downlink massive MIMO,” in Proc. of IEEE International Conference on Communications, pp. 2135-2139, June 10-15, 2012. Article(CrossRef Link).

[6] L. Yang, L. Yang, H. Zhu, and Y. Liang, "Sum rate and Energy Efficiency of Massive MIMO Downlink with Channel Aging in Time Varying Ricean Fading Channel,” KSII Transactions on Internet and Information Systems, vol. 12, no. 3, pp. 1098-1112, March, 2018. Article (CrossRef Link).

[7] S. Zhao, B. Shen, and Q. Hua, “A comparative study of low-complexity MMSE signal detection for massive MIMO systems,” KSII Transactions on Internet and Information Systems, vol. 12, no. 4, pp. 1504-1526, April, 2018. Article (CrossRef Link).

[8] X. Ge, R. Zi, H. Wang, J. Zhang, and M. Jo, "Multi-User Massive MIMO Communication Systems Based on Irregular Antennas Arrays,” IEEE Transactions on Wireless Communications, vol. 15, no. 8, pp. 5287-5301, August 2016. Article(CrossRef Link).

[9] Q. Yu, C. Han, L. Bai, J. Choi, and X. Shen, "Low-Complexity Multiuser Detection in Millimeter-Wave Systems Based on Opportunistic Hybrid Beamforming,” IEEE Transactions on Vehicular Technology, vol. 67, no. 10, pp. 10129-10133, October 2018. Article(CrossRef Link).

[10] L. Bai, T. Li, J. Liu, Q. Yu, and J. Choi, "Large-Scale MIMO Detection Using MCMC Approach With Blockwise Sampling,” IEEE Transactions on Communications, vol. 64, no. 9, pp. 3697-3707, September 2016. Article (CrossRef Link).

[11] S. Wang, Y. Liu, W. Zhang, and H. Zhang, “Achievable Rates of Full-Duplex Massive MIMO Relay Systems Over Rician Fading Channels,” IEEE Transactions on Vehicular Technology, vol. 66, no. 11, pp. 9825-9837, November 2017. Article (CrossRef Link).

[12] L. Lu, G. Y. Li, A. L. Swindlehurst, A. Ashikhmin, and R. Zhang, "An Overview of Massive MIMO: Benefits and Challenges," IEEE International Journal on Selected Topics in Signal Processing, vol. 8, no. 5, pp. 742-758, October, 2014. Article (CrossRef Link).

[13] P. Xu, J. Wang, and J. Wang, "Effect of Pilot Contamination on Channel Estimation in Massive MIMO Systems," in Proc. of IEEE International Conference on Wireless Communication and Signal Processing (WCSP), pp. 1-6, October 24-26, 2013. Article (CrossRef Link) .

[14] J. Jose, A. Ashikhminl, T. L. Marzetta, and S. Vishwanath, "Pilot Contamination and Precoding in Multiuser TDD Systems,” IEEE Transactions on Wireless Communications, vol. 10, no. 8, pp. 2640-2651, August, 2011. Article (CrossRef Link).

[15] C. D. Araujo, T. Maksymyuk, A. L. F. de Almeida, T. Maciel, J. C. M. Mota, and M. Jo, "Massive MIMO: survey and future research topics,” IET Communications, vol. 10, no. 15, pp. 1938-1946, May, 2016. Article (CrossRef Link). 
[16] A. Papazafeiropoulos, S. K. Sharma, and S. Chatzinotas, "Impact of transceiver impairments on the capacity of dual-hop relay massive MIMO systems," in Proc. of IEEE Globecom Workshops, pp. 1-6, December 6-10, 2015. Article (CrossRef Link).

[17] A. Papazafeiropoulos, and T. Ratnarajah, "Downlink MIMO HCNs with residual transceiver hardware impairments,” IEEE Communications Letters, vol. 20, no. 10, pp. 2023-2026, October, 2016. Article (CrossRef Link).

[18] X. Xia, D. Zhang, K. Xu, W. Ma, and Y. Xu, "Hardware impairments aware transceiver for full-duplex massive MIMO relaying,” IEEE Transactions on Signal Processing, vol. 63, no. 24, pp. 6565-6580, December, 2015. Article (CrossRef Link).

[19] E. Bj”ornson, J. Hoydis, M. Kountouris, and M. Debbah, "Massive MIMO systems with non-ideal hardware: Energy Efficiency, Estimation, and Capacity Limits,” IEEE Transactions on Information Theory, vol. 60, no.11, pp. 7112-7139, November, 2014. Article (CrossRef Link).

[20] E. Bjornson, M. Matthaiou, and M. Debbah, "Massive MIMO with non-ideal arbitrary arrays: Hardware scaling laws and circuit-aware design," IEEE Transactions on Wireless Communications, vol. 14, no. 8, pp. 4353-4368, August, 2015. Article (CrossRef Link).

[21] R. Krishnan, M. R. Khanzadi, N. Krishnan, Y. Wu, A. G. i Amat, T. Eriksson, and R. Schober, "Linear massive MIMO precoders in the presence of phase noise-A large scale analysis," IEEE Transactions on Vehicular Technology, vol. 65, no. 5, pp. 3057-3071, May, 2016. Article (CrossRef Link).

[22] A. Pitarokoilis, S. K. Mohammed, and E. G. Larsson, "Uplink performance of time-reversal MRC in massive MIMO systems subject to phase noise," IEEE Transactions on Wireless Communications, vol. 14, no. 2, pp. 711-723, February, 2015. Article (CrossRef Link) .

[23] F. Athley, G. Durisi, and U. Gustavsson, "Analysis of massive MIMO with hardware impairments and different channel models," in Proc. of 9th European Conference on Antennas and Propagation (EuCAP), pp. 1-5, April 12-17, 2015.

[24] R. Corvaja, and A. G. Armanda, "Phase noise degradation in massive MIMO downlink with zero-forcing and maximum ratio transmission," IEEE Transactions on Vehicular Technology, vol. 65, no. 10, pp. 8052-8059, October, 2016. Article (CrossRef Link).

[25] K. A. Papazafeiropoulos, "Impact of general channel aging conditions on the downlink performance of massive MIMO,” IEEE Transactions on Vehicular Technology, vol. 66, no. 2, pp. 1428-1442, February, 2017. Article (CrossRef Link).

[26] J. Zhu, D. W. K. Ng, N. Wang, R. Schober, and V. K. Bhargava, “Analysis and design of secure massive MIMO systems in the presence of hardware impairments," IEEE Transactions on Wireless Communications, vol. 16, no. 3, pp. 2001-2016, March, 2017. Article (CrossRef Link).

[27] A. Papazafeiropoulos, B. Clerckx, and T. Ratnarajah, "Rate-splitting to mitigate residual transceiver hardware impairments in massive MIMO systems," IEEE Transactions on Vehicular Technology ,vol. 66, no. 9, pp. 8196-8211, September, 2017. Article (CrossRef Link).

[28] M. R. Khanzadi, G. Durisi, and T. Eriksson, "Capacity of SIMO and MISO Phase-Noise Channels With Common/Separate Oscillators,” IEEE Transactions on Communications, vol. 63, no.9, pp. 3218-3231, September 2015. Article (CrossRef Link).

[29] A. Kazerouni, F. J. Lopez-Martinez, and A. Goldsmith, "Increasing capacity in massive MIMO networks via small cells,” in proc. of IEEE Globecom Workshop, pp.358-363, December 8-12, 2014. Article (CrossRef Link).

[30] A. Pitarokoilis, S. K. Mohammed, and E. G. Larsson, "Effect of oscillator phase noise on uplink performance of large MU-MIMO systems,” in Proc. of 50th Annual Allerton Conference on Communication, Control and Computing, pp. 1190-1197, October 1-5, 2012.

Article (CrossRef Link). 
[31] M.Wenk, "MIMO-OFDM Testbed: Challenges, Implementations, and Measurement Results," Microelectronics, Konstanz, Germany: Hartung-Gorre, 2010.

[32] T. Marzetta, "Noncooperative cellular wireless with unlimited numbers of base station antennas," IEEE Transactions on Wireless Communications, vol. 9, no. 11, pp. 3590-3600, November, 2010. Article (CrossRef Link) .

[33] X. Yang, and A. O. Fapojuwo, "Performance analysis of hexagonal cellular networks in fading channels," Wireless Communications and Mobile Computing, vol. 16, no. 7, pp. 850-867, 2016. Article (CrossRef Link)

[34] P. Tebe, Y. Kuang, A. E. Ampoma, and K.A. Opare, "Mitigating pilot contamination in massive MIMO using cell size reduction ," IEICE Transactions on Communications, vol. E101-B, no. 5, pp. 1280-1290, May, 2018. Article (CrossRef Link).

[35] “FR Agile Transceiver AD9364 Datasheet,” Analog Devices, Norwood, MA, USA, February, 2014.

[36] P. Tebe, Y. Kuang, and J. Kponyo, "Performance analysis and comparison of ZF and MRT based downlink massive MIMO systems," in Proc. of International Conference on Ubiquitous and Future Networks (ICUFN), pp. 383-388, July 8-11,2014.

[37] B. Hassibi and B. M. Hochwald, "How much training is needed in multiple-antenna links?," IEEE Transactions on Information Theory, vol. 49, no. 4, pp. 951-963, 2003. Article (CrossRef Link).

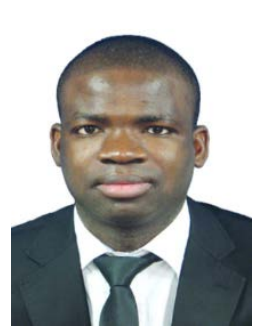

Parfait I. Tebe received the B.Sc. degree in Telecommunication Engineering from Ghana Telecom University College (GTUC), Ghana in 2011, and the M.Sc. degree in Communication and Information Engineering from University of Electronic Science and Technology of China (UESTC), China in 2014. He is currently working towards the PhD degree in Communication and Information Engineering at UESTC. He is a student member of IEEE and IEICE and his current research interests focus on massive MIMO and signal processing in wireless communications.

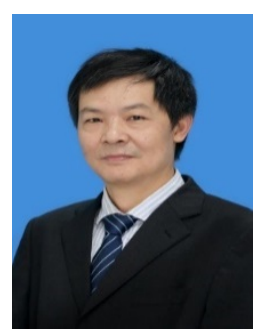

Guangjun Wen received his M.S. degree from Chongqing University of China in 1995 and his PhD degree from University of Electronic Science and Technology of China (UESTC) in 1998. He is currently a professor in UESTC. His research and industrial experience covers a broad spectrum of electromagnetics, including RF, Microwave, Millimeter- wave Integrated Circuits and Systems design for Wireless Communication, Navigation, Identification, Mobile TV applications, RF- IC/MMIC/MMMIC device modeling, RFID system and networks, antennas, as well as model of electromagnetic metamaterial and its application in microwave engineering area, etc. He is a senior member of IEEE and has published several research papers in international conferences and journals. He also holds patents and is author of many books.

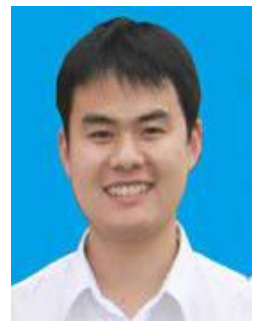

Jian Li received the B.S., MS. and $\mathrm{PhD}$ degrees from the University of Electronic Science and Technology of China, Chengdu, China, in 2007, 2010, and 2015 respectively. He is currently an associate professor with the University of Electronic Science and Technology of China, and was a visiting scholar with the Center for Computational Electromagnetics, Department of Electrical and Computer Engineering, University of Illinois at Urbana-Champaign from 2016 to 2017. His current research interests include RFID, Internet of Things(IoT), Metamaterials/Met surfaces, Applied Electromagnetics and RF, Microwave, Millimeter wave Integrated Circuits and systems, Wireless systems, etc. 


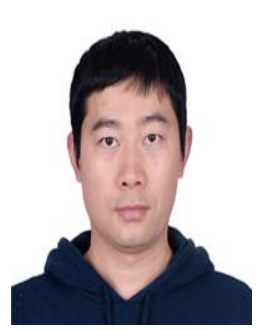

Yongjun Huang received the M.S. degree and Ph.D. agree from the University of Electronic Science and Technology of China (UESTC) in June 2010, and December 2016, respectively. From September 2013 to September 2015, he was a visiting scholar with the Solid-State Science and Engineering, and Mechanical Engineering, Columbia University, and a visiting project scientist with the Electrical Engineering, University of California at Los Angeles (UCLA). He is currently an assistant professor in UESTC since February 2017. He was awarded as the Excellent Doctoral Dissertation Honor selected by China Education Society of Electronics in 2017 and got the support by National Postdoctoral Program for Innovative Talents. He is a member of IEEE and his research interests include the chip-scale photonic crystal cavity opt mechanics, low phase noise RF sources, and high-resolution force/field sensors; and the antennas, microwave passive components, electromagnetic metamaterials, large scale MIMO systems, etc. He has published more than 60 journal papers and more than 60 conference talks.

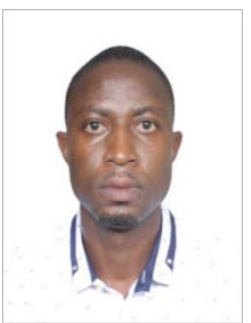

Affum E. Ampoma received the BSc. degree in Electronics and Communication Engineering from Kwame Nkrumah University of Science and Technology (KNUST), Ghana in 2007, the M.Tech degree in Communication Systems from SRM University, India, in 2009. He has just completed the Ph.D. degree in Communication and Information Engineering at University of Electronic Science and Technology of China (UESTC). His current research interests include design and application of multiple antennas for next generation wireless communication, information theory and signal processing.

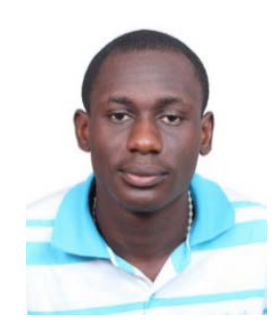

Kwame O. Gyasi received his B.Sc. degree in Telecommunication Engineering from Kwame Nkrumah University of Science and Technology, Kumasi, Ghana in 2011. In 2014, he received his M.Sc. degree in Communication and information Engineering from University of Electronic Science and Technology of China (UESTC). He has just completed the Ph.D. degree in Communication and Information Engineering at UESTC. His research interests include Circularly Polarized antennas, printed wideband antennas, planar monopole antennas and slot antennas. 